\title{
Proper Sample Preparation for Characterization of Drug Delivery Carriers in TEM
}

\author{
Hui Qian ${ }^{1}$
}

${ }^{1 .}$ National Institute for Nanotechnology, NRC, Edmonton, AB, T6G 2M9, Canada

TEM has been most frequently used for the evaluation of morphology, size, composition and ultrastructure of colloidal delivery carriers, such as nanoparticles (NPs), liposomes, lipoproteins and polymer-based colloids [1,2]. Increasingly complex systems have been developed for targeted delivery, sustained release formulations and even drug tracing after administration [3], leading to more challenging sample preparation of these colloidal systems for their study under the TEM. The combination of different sample preparation methods, such as staining, labeling, freeze-fracture and plunge freezing etc., and the corresponding TEM imaging techniques have seen an increase in demand.

Several selected carriers, such as unilamellar and multilamellar liposomes and lipoproteins have been used to demonstrate different sample preparation methods for cryo-TEM analysis. Traditional negative staining, specific site labeling and plunge freezing were applied and the results showed that the morphology and structure of colloidal carriers can be altered by improper sample preparations. Electron tomography has been used to study 3D structure of NP carriers. More accurate reconstructions are achieved using fiducial markers such as colloid Au NPs. A new method of introducing markers for cryo electron tomography will be demonstrated (to be patented) and the selected delivery carrier is core-shell metallic Gd-Au NPs.

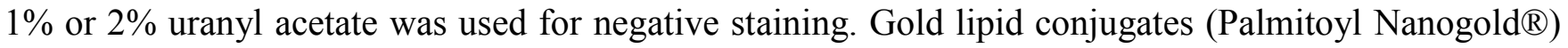
and other labeling agents were purchased from Nanoprobes. Briefly, a $3 \mu \mathrm{l}$ drop of aqueous sample with adjusted concentration was placed on TEM grids which were glow discharged before applying sample solutions. The excess liquid was blotted using filter paper. The samples on grids were either negatively stained with uranyl acetate or labeled with nanogold agents. To obtain the frozen-hydrated specimen embedded in vitreous ice, the samples on holey carbon grids were rapidly plunged into liquid ethane. Then the samples were transferred to a JEOL 2200FS TEM for cryo-TEM analysis. To prepare samples for tomography, $5 \mathrm{~nm}$ to $10 \mathrm{~nm}$ gold NPs were pre-fabricated on film before sample solution being applied to the grid. The tilt series of images for tomography were obtained by tilting specimen from $75^{\circ}$ to $+75^{\circ}$ with $2^{\circ}$ increments.

Normally staining is used to enhance the contrast, but sometimes it can be troublesome. As shown in Fig.1a, negatively stained liposomal vesicles were collapsed after being dried in air. The vesicles that were stained with Au NPs and embedded in vitreous ice also showed shape-change as in Fig.1b. The intact vesicles embedded in vitreous ice are well dispersed with circular shape as expected, shown in Fig. 1c. Fig. 2 shows the effect of the alignment markers in the final reconstructed tomography data.

The arcs as arrow marked in Fig. 2a were shown in the series without alignment markers. While the final 3D reconstructed result more accurately revealed real information of the core-shell structure for the 
specimen with alignment markers as shown in Fig. 2b and 2c. The solid Au NP is surrounded by Gd NP clusters.

Based on experience obtained from many experiments, the realization of requirement for proper sample preparation is very crucial for further analysis in TEM. Also the demand and challenge of preparation combination, such as labeling and plunge freezing, contamination free specimen and grids property and low dose imaging challenge for characterizing colloidal carriers in TEM will be discussed at the meeting.

\section{References}

[1] P. Frederik et al, Methods Enzymol., 391 (2005), pp. 431-448

[2] S. A. Abraham et al, Biochim. Biophys. Acta, 1565 (2002), pp. 41-54

[3] T. Allen et al, Science, 303 (2004), pp. 1818-1822

[4] Great thanks would like to Dr. Bach at EMD Serono Cananda Inc. and Dr. Gu from

Department of Oncology at University of Alberta for samples and discussion.
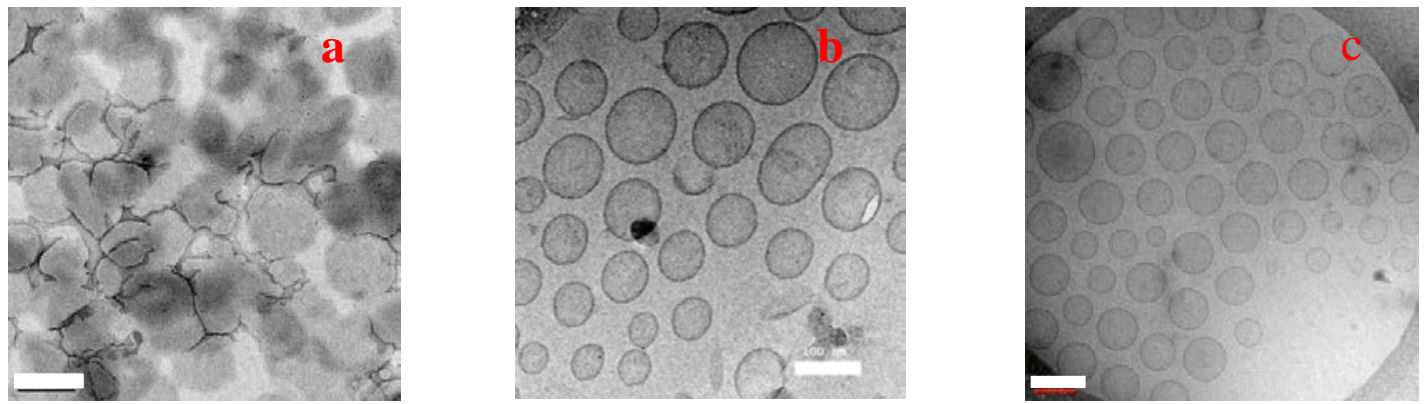

Fig.1: Bright field TEM image of liposomal vesicles a) negatively stained with 1\% uranyl acetate; b) gold lipid conjugates stained and embedded in vitreous ice; c) embedded in vitreous ice. Scale bar is 100 nm.
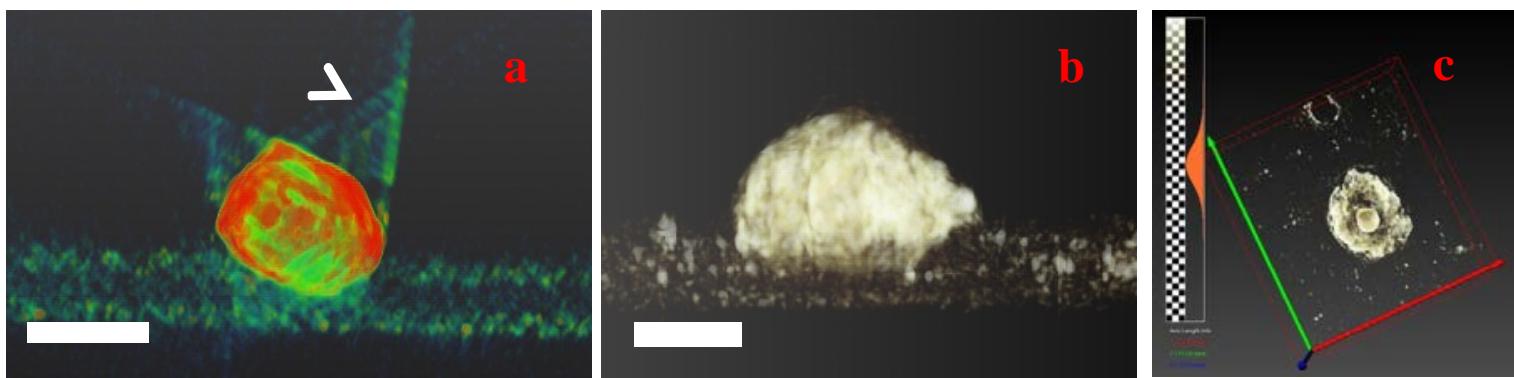

Fig.2: Reconstructed 3D data of Gd-Au NP a) without alignment markers; b) with alignment markers in $\mathrm{X}$ direction view; c) volume view with alignment markers. Scale bar is $30 \mathrm{~nm}$. 